\title{
Efectos comportamentales de un programa de habilidades sociales en jóvenes de 14 a 18 años en situación de vulnerabilidad
}

\author{
Jesús Redondo-Pacheco*, Johan Sebastián Parra-Moreno**, Marianela Luzardo-Briceño***
}

* $\mathrm{PhD}$ en Psicología, Universidad Miguel Hernández de Elche, España. Profesor, Facultad de Psicología, Universidad Pontificia Bolivariana, Bucaramanga, Colombia.

Correo electrónico:

jesus.redondo@upb.edu.co

** Psicólogo. Estudiante investigador, Universidad Pontificia Bolivariana, Bucaramanga, Colombia. Correo electrónico: josepa2414@hotmail.com

*** PhD en Estadística, Universidad de los Andes de Mérida, Venezuela. Profesora, Facultad de Ingeniería Industrial, Universidad Pontificia Bolivariana, Bucaramanga, Colombia. Correo electrónico: marianela.luzardo@upb.edu.co

Recibido: 20 de febrero del 2015 Aprobado: 12 de junio del 2015

Cómo citar este artículo: Redondo-Pacheco, J., Parra-Moreno, J. S. y Luzardo-Briceño, M. (2015). Efectos comportamentales de un programa de habilidades sociales en jóvenes de 14 a 18 años en situación de vulnerabilidad. Pensando Psicología, 11(18), 45-58. doi: http://dx.doi.org/10.16925/ pe.v11i18.1003

\section{Resumen}

Introducción: las habilidades sociales son importantes para la adaptación de los individuos a los diferentes contextos sociales. Para los adolescentes puede ser difícil establecer y mantener interacciones sociales competentes debido a los cambios producto de su desarrollo. Objetivo: analizar los efectos comportamentales de un programa de habilidades sociales en jóvenes de 14 a 18 años en situación de vulnerabilidad, de la Fundación Club Campestre de Bucaramanga. Metodología: para la medición de las habilidades sociales, se utilizó la Escala de Habilidades Sociales (EHS), creada en el 2000 por Elena Gismero en la Universidad Pontificia de Madrid. Se trata de un cuestionario compuesto por 33 ítems que explora la conducta habitual del sujeto en situaciones concretas y valora hasta qué punto las habilidades sociales modulan estas actitudes. Participaron 50 jóvenes pertenecientes a la Fundación Club Campestre, los cuales se dividieron en dos grupos: experimental y control. La metodología de trabajo fue una aplicación de pruebas pre-test para la medición de las habilidades, se continuó con la aplicación del programa y se finalizó con la aplicación del pos-test para el análisis de los resultados. Resultados: el programa generó efectos comportamentales en los jóvenes, es decir, conductas socialmente habilidosas y el reforzamiento de su repertorio de habilidades sociales, presentando mejora en la convivencia en su contexto social, menos respuestas agresivas y más esquemas de respuesta asertiva. Conclusión: los resultados sugieren que los programas de habilidades sociales son herramientas útiles para la intervención de jóvenes con dificultades en el desarrollo de estas.

Palabras clave: convivencia, habilidades sociales, jóvenes, padres. 


\title{
Behavioral Effects of a Social Skills Program among Young People 14 to 18 Years of Age in a Situation of Vulnerability
}

\begin{abstract}
Introduction: Social skills are important for adaptation by individuals to different social contexts. It can be difficult for adolescents to establish and maintain competent social interactions due to changes stemming from their development. Objective: To analyze the behavioral effects of a social skills program among young people 14 to 18 years of age in a situation of vulnerability, at the Fundación Club Campestre de Bucaramanga (country club). Methodology: To measure social skills, the Social Skills Scale (Escala de Habilidades Sociales (EHs)), created in 2000 by Elena Gismero at the Universidad Pontificia de Madrid, was used. It is a 33 item questionnaire that explores the individual conduct of the subject in specific situations and assesses the extent to which social skills modulate these attitudes. 50 students from the Fundación Club Campestre took part and were divided into two groups: experimental and control. The working mythology was the application of pre-tests to measure skills, continued with application of the program and ended with the post-test to analyze the results. Results: The program generated behavioral effects among the young people, in other words, socially skillful conducts in the young people and strengthening of their repertoire of social skills, with improvement in coexistence in their social context, fewer aggressive responses and more assertive response schemes. Conclusion: The results suggest that social skills programs are useful tools for intervention among young people with difficulties developing such skills.
\end{abstract}

Keywords: coexistence, social skills, young people, parents.

\section{Efeitos comportamentais de um programa de habilidades sociais em jovens de 14 a 18 anos em situação de vulnerabilidade}

\section{Resumo}

Introdução: as habilidades sociais são importantes para a adaptação dos indivíduos aos diferentes contextos sociais. Para os adolescentes, pode ser difícil estabelecer e manter interações sociais competentes devido às mudanças produto de seu desenvolvimento. Objetivo: analisar os efeitos comportamentais de um programa de habilidades sociais em jovens de 14 a 18 anos em situação de vulnerabilidade, da Fundación Club Campestre de Bucaramanga (Colômbia). Metodologia: para a medição das habilidades sociais, utilizou-se a Escala de Habilidades Sociais (EHS), criada em 2000 por Elena Gismero, na Universidad Pontificia de Madrid. Trata-se de um questionário composto por 33 itens que explora o comportamento habitual do sujeito em situações concretas e avalia até que ponto as habilidades sociais forjam essas atitudes. Participaram 50 jovens pertencentes à Fundação, os quais foram divididos em dois grupos: experimental e controle. A metodologia de trabalho foi uma aplicação de provas pré-teste para a medição das habilidades, continuou-se com a aplicação do programa e finalizou-se com a aplicação do pós-teste para a análise dos resultados. Resultados: o programa gerou efeitos comportamentais nos jovens, isto é, apresentou melhoras na convivência em seu contexto social, menos respostas agressivas e mais esquemas de resposta assertiva. Conclusão: os resultados sugerem que os programas de habilidades sociais são ferramentas úteis para a intervenção de jovens com dificuldades no desenvolvimento destas.

Palavras-chave: convivência, habilidades sociais, jovens, pais. 


\section{Introducción}

El presente trabajo tiene como finalidad analizar los efectos comportamentales de un programa de habilidades sociales en jóvenes de 14 a 18 años en situación de vulnerabilidad de la Fundación Club Campestre de Bucaramanga. La población de estudio está conformada por 50 jóvenes pertenecientes a la mencionada fundación. Se entiende por personas en situación de vulnerabilidad "aquellas personas que se encuentran en mayor medida expuestos a la exclusión, la pobreza y los efectos de la inequidad y la violencia de todo orden" (Ministerio de Educación Nacional, 2005, p. 5). Además de presentar patrones de relación familiar inadecuados, pueden proceder de familias desestructuradas con muy bajos niveles socioculturales y económicos.

$\mathrm{Al}$ analizar el déficit de habilidades sociales en los jóvenes de la fundación se propone un programa de habilidades sociales que genere un cambio comportamental en sus interacciones sociales. La importancia de este programa radica en cómo, al formar jóvenes con habilidades sociales, se posibilita la construcción de un adulto que puede mantener una convivencia pacífica con los otros, considerando que las habilidades sociales son básicas y un requisito fundamental para la adaptación a la vida social.

Existen diferentes investigaciones que señalan la importancia de las habilidades sociales para la adaptación de los individuos a los diferentes contextos sociales favoreciendo sus relaciones interpersonales (Zambrano, Muñoz y González, 2012; FernándezDaza y Fernández-Parra, 2013), así como en la prevención de trastornos psicopatológicos (Betina y Contini, 2011).

Las interacciones sociales eficaces son necesarias para el ajuste emocional y conductual, un funcionamiento exitoso tanto en el hogar como en la escuela, y para el trabajo. En los adolescentes puede ser especialmente difícil establecer y mantener interacciones sociales competentes debido a los cambios que se producen en su desarrollo y que están asociados a la pubertad. Las interacciones sociales y las relaciones se vuelven cada vez más complejas, pues desempeñan estas relaciones con los amigos un papel crítico en el desarrollo de habilidades sociales y de sentimientos de competencia personal, aspectos fundamentales para el funcionamiento apropiado en la adultez (Phillips y Zigler, 1961; La Greca y López, 1998; Méndez, Inglés y Hidalgo, 2002; Redondo y Inglés, 2009).
Los citados cambios asociados al desarrollo y la evolución de las expectativas sociales que se producen durante la adolescencia pueden hacer que sea especialmente difícil para los jóvenes establecer y mantener interacciones sociales idóneas. Así, dificultades interpersonales en el adolescente pueden tener consecuencias negativas para este. En primer lugar, porque influyen en el rendimiento académico, ya que el adolescente participa poco en clase, tiene muchas dificultades para presentar trabajos en público y, ante posibles dudas, evita preguntar al profesor (Lawrence y Bennet, 1992; Inglés, 2009; Rao et al., 2007). En segundo lugar, al eludir las relaciones con sus iguales se presenta aislamiento y sentimientos de soledad (Walters y Inderbitzen, 1998; Inglés, Méndez y Hidalgo, 2001), y la subsecuente baja tasa de reforzamiento social lleva a la depresión (Francis, Last y Strauss, 1992; Inglés et al., 2010). Por último, los déficits de habilidades sociales llevan a relaciones disfuncionales con los padres, lo cual impide una buena comunicación, la negociación y la resolución efectiva de conflictos (Openshaw, Mills, Adams y Durso, 1992; Martínez, Inglés, Piqueras y Ramos, 2010). Además, aumenta el riesgo de consumo de drogas legales e ilegales (Clark y Kirisci, 1996; Inglés et al., 2007).

La investigación sobre el entrenamiento en habilidades sociales con adolescentes comenzó a mediados de la década de los setenta y ha mejorado mucho con los años, desplazándose más allá de un enfoque centrado en la evaluación y adquisición de las habilidades básicas, hacia técnicas diseñadas para evaluar y promover la generalización y el mantenimiento de un repertorio interpersonal eficaz en situaciones reales y cotidianas. Los primeros trabajos en este campo - en su mayoría - están centrados en las conductas agresivas en jóvenes en ámbitos hospitalarios o residenciales, y su objetivo principal es la intervención en habilidades asertivas (Arkowitz, Hinton, Perl y Himadi, 1978; Bradlyn et al., 1983). Posteriormente, se empezó a incursionar en otras potenciales poblaciones objeto de estudio, como por ejemplo, prevención de la violencia (Meyer y Farrell, 1998); personas con problemas emocionales (Schloss, Schloss y Harris, 1984; Plienis et al., 1987); ansiedad heterosocial (Arkowitz et al., 1978; Schinke, Blythe y Gilchrist, 1981; Conger y Conger, 1982); abuso de sustancias (Englander-Golden, Elconin y Satir, 1986; Gilchrist, Schinke, Trimble y Cvetkovich, 1987) y discapacidades físicas o de desarrollo (Lemanek, Williamson, Gresham y Jensen, 1986). 
La definición de las habilidades sociales es compleja en razón a que estas son muy subjetivas y se caracterizan por ser aprendidas mediante la observación, la imitación y el ensayo-error. Por lo tanto, estas habilidades incluyen comportamientos tanto verbales como no verbales, específicos como discretos, y además se refuerzan con las respuestas positivas del propio medio social, es decir, son recíprocas.

Fue Salter (1949) con su trabajo de terapia de reflejos condicionados, el que acuñó el primer término "personalidad excitatoria". Más tarde, Wolpe (1958) introdujo la expresión "conducta asertiva", la cual se acerca de forma significativa a la terminología actual. Más tarde, Lazarus y Folkman (1971) lo expresaron como "efectividad personal", mientras Liberman (1975) destacaba la personalidad asertiva, aunque no tuvieron mucha transcendencia en la teoría sobre las habilidades sociales.

En general, y de acuerdo con autores más representativos como Milchelson, Sugai, Wood y Kazdin (1987), "las habilidades sociales son consideradas como un conjunto de comportamientos interpersonales complejos" (p. 7). Según la definición anterior, si estas habilidades son "apropiadas o «buenas», la resultante es una mayor satisfacción personal e interpersonal, tanto a corto como a largo plazo" (p. 5).

En este sentido, no se ha podido llegar a un concepto único. Por eso, Michenbaum, Butler y Gruson (1981) afirman que "es imposible desarrollar una definicion consistente de competencia social puesto que esta es parcialmente dependiente del contexto" (p. 34), mientras que otros autores proponen que para estudiar las habilidades sociales, se deben primero reconocer ciertos tipos de comportamientos en los adolescentes (Camacho, 2005).

Así, es la familia el primer y más importante agente socializador en el que se aprenden las destrezas sociales, actitudes y habilidades necesarias para adaptarse al medio en el que se vive. Aunque la mayoría de los niños se convierten en adultos bien adaptados, muchos otros se encuentran con diversos problemas que varían en su forma y gravedad (inadaptación personal y social, problemas psicológicos, etc.). En los primeros años, los niños adquieren destrezas sociales. Los valores y las competencias comienzan a formarse a partir, fundamentalmente, de la observación y de la imitación de sus padres, estimulando o inhibiendo diferentes comportamientos dependiendo de los estilos de crianza (Moore, 1997; Arvelo, 2003; Gracia, Lila y Musitu, 2005; Oliva, Parra y Arranz, 2008; Pichardo, Justicia y Cabezas, 2009; Cuervo, 2010; Peña, Maiquez y Rodrigo, 2014).
En este sentido, diferentes resultados de investigaciones realizadas con poblaciones latinoamericanas confirman la influencia de las condiciones de crianza en niños y adolescentes, sobre sus habilidades sociales y problemas emocionales (Martínez, Rosete yRíos, 2007; González, Valdés, Domínguez, Palomary González, 2008; Rocha, 2003; Solís y Montoya, 2007; González y Méndez, 2006; González, Ampudia y Guevara, 2012).

Todo esto ha llevado al desarrollo de investigaciones que permitan probar la efectividad del entrenamiento en habilidades sociales como instrumento para resolver diversos problemas psicológicos, tales como ansiedad y fobia social (Spence et al., 2000; Inglés, 2009); rechazo social (Sim et al., 2006); conductas desadaptadas y violencia (Nitkowski, Petermann, Büttner, Krause y Petermann, 2009), así como conducta antisocial, delincuencia y adicciones (Savidge et al., 2004). La mayoría de estos instrumentos han demostrado su efectividad en la adquisición, generalización y mantenimiento de las conductas positivas entrenadas, así como para la disminución de conductas problema (Dib y Sturmey, 2007). Los apartados con mejores resultados en los programas de intervención son los entrenamientos en habilidades sociales básicas y avanzadas, más entrenamientos para la solución de problemas y reestructuración cognoscitiva a través de diferentes técnicas tales como las instrucciones verbales, modelamiento, juego de roles, retroalimentación, reforzamiento y tareas (Beelmann y Lösel, 2006; Caballo, 2009; Sukhodolsky et al., 2005).

En esta investigación, en la totalidad de los jóvenes participantes no está presente una de las figuras paternas, y se evidencia una falencia en el desarrollo de sus habilidades sociales. Así, se planteó un programa de habilidades sociales a fin de generar en ellos un aprendizaje y modificación de las conductas que están dentro del repertorio de las habilidades sociales, por medio de talleres psicoeducativos utilizando estrategias de aprendizaje tales como la imitación, observación o instrucción, en las que se analizaron los efectos comportamentales de un programa de habilidades sociales en los jóvenes participantes.

En conformidad con todo lo expuesto anteriormente, el propósito del presente estudio fue analizar los efectos comportamentales de un programa de habilidades sociales en jóvenes de 14 a 18 años en situación de vulnerabilidad, de la Fundación Club Campestre de Bucaramanga. Esto a través de una identificación previa de sus habilidades sociales (pre-test), la posterior aplicación del programa de 
habilidades sociales y, finalmente, la determinación de las habilidades sociales (pos-test) después del citado programa. Así, esta investigación plantea una hipótesis principal: En el grupo que recibe el programa de entrenamiento en habilidades sociales existirán diferencias significativas entre las puntuaciones pre-test y pos-test obtenidas en la escala EHS, mientras que no existirán estas diferencias significativas en el grupo control.

\section{Metodología}

\section{Participantes}

En el estudio participaron 50 jóvenes, entre los 14 y los 18 años, con una edad media de 15,66 años y una desviación típica de 1,07. De género masculino y en situación de vulnerabilidad, se desempeñan como caddies de tenis beneficiarios de la Fundación Club Campestre de Bucaramanga. Fueron asignados mediante un muestreo no probabilístico intencional: 25 jóvenes asisten en la jornada de la mañana como grupo experimental, y 25 jóvenes asisten en la tarde como el grupo control. Cabe indicar que no se presentó grado de mortalidad experimental tras la aplicación del programa de EHS. La tabla 1 muestra la distribución de los sujetos por grupo y edad.

\section{Instrumentos}

\section{La Escala de Habilidades Sociales (EHS)}

Creada en el 2000 por Elena Gismero-Gonzáles en la Universidad Pontificia de Madrid y adaptada por César Ruiz-Alba. Se trata de un cuestionario compuesto por 33 ítems que exploran la conducta

Tabla 1

Número (y porcentaje) de sujetos de la población total clasificados por grupo y por edad

\begin{tabular}{ccccccc}
\hline Edad & & & & & & \\
\hline \multicolumn{1}{c}{ Grupo } & $\mathbf{1 4}$ & $\mathbf{1 5}$ & $\mathbf{1 6}$ & $\mathbf{1 7}$ & $\mathbf{1 8}$ & Total \\
\hline \multirow{2}{*}{ Experimental } & 4 & 5 & 12 & 3 & 1 & 25 \\
& $(16 \%)$ & $(20 \%)$ & $(48 \%)$ & $(12 \%)$ & $(4 \%)$ & $(100 \%)$ \\
Control & 5 & 6 & 8 & 5 & 1 & 25 \\
& $(20 \%)$ & $(24 \%)$ & $(32 \%)$ & $(20 \%)$ & $(4 \%)$ & $(100 \%)$ \\
\hline \multirow{2}{*}{ Total } & 9 & 11 & 20 & 8 & 2 & 50 \\
& $(36 \%)$ & $(44 \%)$ & $(80 \%)$ & $(32 \%)$ & $(8 \%)$ & $(100 \%)$ \\
\hline
\end{tabular}

Nota. Elaboración propia. habitual del sujeto en situaciones concretas y valora hasta qué punto las habilidades sociales modulan estas actitudes. Su aplicación puede ser individual o colectiva; las edades de aplicación son para adolescentes a partir de los 12 años y adultos, y el tiempo de duración de la aplicación de la prueba de 15 a 20 minutos.

Las escalas de evaluación son: (a) Autoexpresión en situaciones sociales; (b) Defensa de los propios derechos como consumidor; (c) Expresión de enfado o disconformidad; (d) Decir no y cortar interacciones; (e) Hacer peticiones con el sexo opuesto; (f) Iniciar interacciones positivas con el sexo opuesto.

Los ítems presentan una adecuada fiabilidad en términos de consistencia interna, tanto por factores, como en la escala total, lo que facilita su aplicación en diversos contextos. La consistencia interna de los 30 reactivos fue de 0,84 ; de la dimensión académica/ laboral, 0,89; de la social, 0,73 ; de la emocional, 0,73 ; de la familiar, 0,80; y del físico, 0,78.

La validez de esta estructura multidimensional definida a priori se ha constatado empíricamente con análisis factoriales exploratorios en muestras de España, lo cual permite concluir que la escala tiene una buena calidad psicométrica. La muestra empleada para el análisis correlacional fue llevada a cabo con 770 adultos y 1015 jóvenes, respectivamente. Todos los índices de correlación superan los factores encontrados en el análisis factorial de los elementos en la población general, según Gismero (por ejemplo, 0,74 entre los adultos y 0,70 entre los jóvenes).

La confiabilidad del instrumento muestra una consistencia interna alta, como se expresa en su coeficiente de confiabilidad $\alpha=0,88$, el cual se considera elevado, pues supone que el $88 \%$ de la varianza de los totales se debe a lo que los ítems tienen en común (habilidades sociales o asertividad).

\section{Ficha sociodemográfica}

Diseñada por los investigadores, recoge información concreta de variables sociodemográficas tales como el estrato socioeconómico, el sexo, la edad, el nivel de escolaridad, así como las personas con las que viven actualmente los sujetos y el lugar que ocupan dentro de sus hermanos, entre otros datos.

\section{Talleres sobre habilidades sociales}

Los talleres que se llevaron a cabo con los jóvenes se presentan en la tabla 2. 
Tabla 2

Talleres realizados con el grupo experimental

\begin{tabular}{|c|c|}
\hline Taller N. ${ }^{\circ} 1$ & Introducción \\
\hline Objetivo & $\begin{array}{l}\text { Dar a conocer el programa de habilidades sociales } \\
\text { a los jóvenes de la fundación. }\end{array}$ \\
\hline Población & Grupo experimental y grupo control \\
\hline Metodología & $\begin{array}{l}\text { La presentación del programa se realiza según } \\
\text { la jornada de cada adolescente; se presenta una } \\
\text { explicación detallada sobre el objetivo, la justifi- } \\
\text { cación de lo que se quiere lograr con esta activi- } \\
\text { dad. Se responden las preguntas de los jóvenes, se } \\
\text { toman sugerencias sobre los diferentes talleres y } \\
\text { las posibles actividades lúdicas a realizar. }\end{array}$ \\
\hline
\end{tabular}

Duración $\quad 45$ a 60 minutos.

\begin{tabular}{|c|c|}
\hline Taller N. ${ }^{\circ} 2$ & Espacio personal \\
\hline Objetivo & $\begin{array}{l}\text { Identificar la proximidad-distancia entre dos o } \\
\text { más personas cuando se comunican. }\end{array}$ \\
\hline Población & Grupo experimental \\
\hline Metodología & $\begin{array}{l}\text { Los talleres se realizan a los jóvenes pertenecien- } \\
\text { tes al grupo experimental y se inicia con ejercicios } \\
\text { de dos tipos: } \\
\text { Proximidad: se les pide a los jóvenes que se ubi- } \\
\text { quen en el salón de tal forma que queden dos } \\
\text { jóvenes en un mismo puesto durante un tiempo } \\
\text { de } 10 \text { minutos, con el fin de que no tengan un } \\
\text { espacio personal entre ellos. } \\
\text { Distancia: en este ejercicio, por el contrario, se le } \\
\text { pide a los jóvenes que se ubiquen en todo el espa- } \\
\text { cio del salón, separados los unos de los otros, con } \\
\text { el fin de denotar que existe una distancia mínima } \\
\text { para la interacción de la personas. } \\
\text { Se procede a dar la explicación de los ejercicios. }\end{array}$ \\
\hline Duración: & 45 a 60 minutos. \\
\hline
\end{tabular}

\begin{tabular}{|c|c|}
\hline Taller N. ${ }^{\circ} 3$ & Inteligencia emocional \\
\hline Objetivo & $\begin{array}{l}\text { Analizar los estilos de respuesta emocional en } \\
\text { situaciones reales de juego. }\end{array}$ \\
\hline Población & Grupo experimental \\
\hline Metodología & $\begin{array}{l}\text { El taller consiste en un partido de futbol, en el } \\
\text { cual se crean situaciones reales de respuesta ante } \\
\text { situaciones aversivas, dándoles las siguientes indi- } \\
\text { caciones: } \\
\text { 1. Formar dos grupos con un mismo número de } \\
\text { integrantes. } \\
\text { 2. Cuando suene el silbato deben pasar al centro } \\
\text { de la cancha. } \\
\text { 3. Analizar el tipo de situación social que se dio. } \\
\text { 4. Generar posibles alternativas de respuesta } \\
\text { emocional. } \\
\text { Se concluye con una breve explicación teórica } \\
\text { sobre la inteligencia emocional. }\end{array}$ \\
\hline Duración & 45 a 60 minutos. \\
\hline
\end{tabular}

\begin{tabular}{|c|c|}
\hline Taller N. ${ }^{\circ} 4$ & Asertividad \\
\hline Objetivo & $\begin{array}{l}\text { Crear la habilidad de respuesta para actuar en el } \\
\text { momento adecuado. }\end{array}$ \\
\hline Población & Grupo experimental \\
\hline Metodología & $\begin{array}{l}\text { Se inicia con una charla sobre el tema de la aser- } \\
\text { tividad; después se realiza un juego de roles en } \\
\text { el que se dan situaciones sociales a los jóvenes y } \\
\text { ellos deben generar una puesta en escena en la } \\
\text { cual encuentren respuestas asertivas a la situación } \\
\text { propuesta. } \\
\text { Para finalizar se lleva a cabo una socialización del } \\
\text { tema. }\end{array}$ \\
\hline Duración & 45 a 60 minutos. \\
\hline
\end{tabular}

\begin{tabular}{ll}
\hline Taller N. ${ }^{\circ}$ & Discernimiento \\
\hline Objetivo & $\begin{array}{l}\text { Identificar qué aspectos de la vida son producti- } \\
\text { vos para la adaptación al medio. }\end{array}$ \\
Población & Grupo experimental \\
Metodología & $\begin{array}{l}\text { Se les solicita a los jóvenes que se dividan en cinco } \\
\text { grupos de cinco personas. Se les da un paquete }\end{array}$ \\
& $\begin{array}{l}\text { con cinco preguntas, cada pregunta con dos alter- } \\
\text { nativas de respuestas. Se promedian los resultados } \\
\text { de cada grupo y se obtiene una clasificación. }\end{array}$ \\
& $\begin{array}{l}\text { Para finalizar se retroalimenta el resultado obte- } \\
\text { nido por cada grupo de jóvenes. }\end{array}$ \\
& 45 a 60 minutos.
\end{tabular}

Duración: $\quad 45$ a 60 minutos.

\begin{tabular}{|c|c|}
\hline Taller N. ${ }^{\circ} 6$ & Manejo de agresividad \\
\hline Objetivo & $\begin{array}{l}\text { Mostrar las diferentes alternativas de respuestas } \\
\text { no agresivas. }\end{array}$ \\
\hline Población & Grupo experimental \\
\hline Metodología & $\begin{array}{l}\text { Se lleva a cabo una feria de la agresividad en forma } \\
\text { circular, iniciando desde la situación mínima, } \\
\text { como una mirada, hasta llegar a la más fuerte, la } \\
\text { muerte. Primero los jóvenes ingresan al kiosco y } \\
\text { cada uno va observando el puesto de agresividad } \\
\text { en forma ordenada. Luego se les da una serie de } \\
\text { palabras para ubicarlas dentro del círculo donde } \\
\text { ellos consideren que deben ir. } \\
\text { Después se realiza una explicación teórica y una } \\
\text { socialización sobre el manejo de la agresividad. }\end{array}$ \\
\hline
\end{tabular}

Duración $\quad 45$ a 60 minutos.

\begin{tabular}{ll}
\hline Taller N. ${ }^{\circ} 7$ & Hacer y recibir cumplidos \\
\hline Objetivo & Aprender a realizar y hacer cumplidos. \\
Población & Grupo experimental \\
Metodología & $\begin{array}{l}\text { Se hacen diferentes micro-competencias para que } \\
\text { cada uno tenga la oportunidad de hacer y recibir } \\
\text { halagos. }\end{array}$ \\
Duración & 45 a 60 minutos. \\
\hline
\end{tabular}


(viene)

\begin{tabular}{ll}
\hline Taller N. ${ }^{\circ} 8$ & Habilidades interpersonales \\
\hline Objetivo & Generar habilidades de interacción social. \\
Población & Grupo experimental \\
Metodología & $\begin{array}{l}\text { Se forman cinco grupos de cinco jóvenes que son } \\
\text { seleccionados por el moderador; se les entregan } \\
\text { los materiales necesarios para construir una edi- } \\
\text { ficación (todos tendrán los mismos materiales } \\
\text { y \$1.00 en efectivo), teniendo en cuenta cuatro } \\
\text { factores: altura, estabilidad, costo y trabajo en } \\
\text { equipo, con la posibilidad de compra de más } \\
\text { materiales vendidos por el moderador del taller. }\end{array}$ \\
& $\begin{array}{l}\text { Se finaliza eligiendo al ganador y con un cierre } \\
\text { sobre el tema. }\end{array}$ \\
& $\begin{array}{l}\text { 45 a } 60 \text { minutos. } \\
\text { Duración }\end{array}$ \\
\hline Taller N. ${ }^{\circ 9}$ & Empatía \\
\hline Objetivo & $\begin{array}{l}\text { Resaltar la importancia de entender y compren- } \\
\text { der al otro. }\end{array}$ \\
Población & Grupo experimental \\
Metodología & $\begin{array}{l}\text { Cine-foro con la película A prueba de fuego. } \\
\text { Al finalizar la película se socializa. }\end{array}$ \\
Duración & 45 a 60 minutos. \\
\hline
\end{tabular}

\begin{tabular}{|c|c|}
\hline Taller N. ${ }^{\circ} 10$ & Toma de decisiones \\
\hline Objetivo & $\begin{array}{l}\text { Orientar a los jóvenes acerca de las decisiones que } \\
\text { tomen. }\end{array}$ \\
\hline Población & Grupo experimental \\
\hline Metodología & $\begin{array}{l}\text { Se lleva a cabo un foro en el que interactúan el } \\
\text { moderador y los jóvenes, haciendo y dando res- } \\
\text { puestas a las inquietudes del grupo en general. }\end{array}$ \\
\hline Duración & 45 a 60 minutos. \\
\hline
\end{tabular}

Nota. Elaboración propia.

\section{Procedimiento}

Se llevó a cabo una entrevista con el director de la fundación, así como con los jóvenes, en la cual se le explicaron los beneficios de participar en el estudio y la libertad de abandonar el mismo si lo deseaban. Posteriormente, los tutores legales firmaron el consentimiento informado.

Los 25 jóvenes que asisten en la jornada de la mañana fueron asignados al grupo experimental y los 25 jóvenes que asisten en la tarde al grupo control. Se aplicó la Escala de Habilidades Sociales en el pretest a todos los participantes. Al terminar, se aplicó el programa de habilidades sociales, y al finalizar dicha aplicación se llevó a cabo una nueva aplicación de la ESH (pos-test), con el fin de evaluar los efectos del programa de habilidades sociales.
El programa se llevó a cabo durante cuatro meses, con una duración intensiva de tres talleres por semana, durante 30 a 45 minutos por cada día de taller. Dentro del programa de habilidades sociales se desarrollaron actividades satélites para el desarrollo integral del programa (tardes deportivas, salida a cine, salidas a centros recreativos).

\section{Resultados}

Este trabajo aporta algunos datos a la investigación sobre los efectos comportamentales de un programa de habilidades sociales en jóvenes.

\section{Comparación antes de la aplicación del programa de intervención}

Con el fin de analizar las diferencias en el grupo control en el pre-test y pos-test de la EHs, se aplicó la prueba de significación estadística t de Student, en la cual se comparan las medias y desviaciones estándar de grupos de datos y se determina si entre esos parámetros las diferencias son estadísticamente significativas. Aunque el tamaño de la población no es muy grande, se incluyó el índice $d$ (diferencia media tipificada) propuesto por Cohen (1988), el cual permite valorar la magnitud o el tamaño del efecto de las diferencias halladas. Este índice se calcula mediante el cociente entre la diferencia de las puntuaciones medias de los grupos y el error típico de estimación. Su interpretación es la siguiente: tamaño del efecto pequeño/bajo $(0,20-0,49)$, moderado $(0,51-0,79)$, y alto/grande $(d \geq 0,80)$.

La tabla 2 muestra las puntuaciones medias y desviaciones típicas de los jóvenes en las seis escalas y la total de la EHs. En cuanto al grupo control, se apreciaron mínimas variaciones en la diferencias de media general, alcanzando una media de 30 en la evaluación pre-test y 28,6 en la evaluación pos-test. Así mismo, la diferencia de medias por escalas del EHS, respecto al pre-test y al pos-test, mostró un mínimo incremento en las escalas Autoexpresión en situaciones sociales, Decir no y cortar interacciones, y Hacer peticiones. Por el contrario, en las escalas Defensa de los propios derechos como consumidor, Expresión de enfado o disconformidad, e Iniciar interacciones positivas con el sexo opuesto, así como en la puntuación total, se aprecia que la diferencia de medias es menor, siendo la magnitud de estas diferencias pequeña, excepto en la Escala II (Defensa de los propios derechos 
como consumidor) y la Escala vi (Iniciar interacciones positivas con el sexo opuesto), en las cuales el tamaño del efecto fue muy grande (Cohen, 1988).

Los integrantes del grupo experimental fueron sometidos a un programa de intervención con el fin de optimizar las habilidades sociales durante cuatro meses, con una duración intensiva de tres talleres por semana de 30 a 45 minutos cada día de taller.

Comparando el grupo experimental antes de participar en el programa de intervención de habilidades sociales y después de la aplicación del mismo, se puede apreciar un incremento en el promedio general y en todas las escalas del EHs evaluadas. El grupo experimental mostró una considerable diferencia de media general, alcanzando una media de 28,40 en el pre-test y 86,64 en el pos-test, de manera que es una diferencia positiva a favor de los participantes del grupo experimental al concluir el programa de intervención. Del mismo modo, los resultados respecto a las diferentes escalas de la EHs indican que los jóvenes del grupo experimental puntúan significativamente más alto en el pos-test que en el pre-test en todas las escalas, así como en la puntuación total, en este caso la magnitud de las diferencias fue muy grande (Cohen, 1988; ver tabla 3).

\section{Comparación de los resultados después de la aplicación del programa de intervención}

La tabla 4 muestra la comparación de las medias por escalas y total del grupo control y experimental en el pos-test (promedio de 28,69 para el grupo control y 86,64 grupo experimental).

Tabla 3

Medias y (desviaciones típicas) del grupo control en el pre-test y pos-test de la EHS

\begin{tabular}{lcccccc}
\hline \multicolumn{1}{c}{ Escalas } & Pre-test & Pos-test & & & \\
\cline { 2 - 6 } & $\begin{array}{c}\text { Media y } \\
\text { desviación típica }\end{array}$ & $\begin{array}{c}\text { Media y } \\
\text { desviación típica }\end{array}$ & $t$ & $p$ & $d$ Cohen \\
\hline Autoexpresión en situaciones sociales (I) & $29,48(26,21)$ & $31,04(26,67)$ & $-0,41$ & 0,68 & $-0,063$ \\
Defensa de los propios derechos como consumidor (II) & $28,36(18,57)$ & $26,16(16,59)$ & 0,84 & 0,40 & 0,125 \\
Expresión de enfado o disconformidad (III) & $47,16(28,97)$ & $45,36(29,32)$ & 0,49 & 0,62 & 0,062 \\
Decir no y cortar interacciones (IV) & $38,84(28,30)$ & $41,64(28,93)$ & $-0,32$ & 0,74 & $-0,098$ \\
Hacer peticiones (v) & $33,12(30,67)$ & $33,64(30,23)$ & $-0,52$ & 0,60 & $-0,017$ \\
Iniciar interacciones positivas con el sexo opuesto (VI) & $46,36(23,82)$ & $40,60(22,46)$ & 0,16 & 0,86 & 0,248 \\
PDG & $30(22,40)$ & $28,60(20,33)$ & 0,28 & 0,78 & 0,065 \\
\hline
\end{tabular}

Nota. Elaboración propia.

Tabla 4

Medias y (desviaciones típicas) del grupo experimental en el pre-test y pos-test de la EHS

\begin{tabular}{lccccc}
\hline & \multicolumn{1}{c}{ Pre-test } & Pos-test & & \\
\cline { 2 - 5 } \multicolumn{1}{c}{ Escalas } & $\begin{array}{c}\text { Mediay } \\
\text { desviación típica }\end{array}$ & $\begin{array}{c}\text { Media y } \\
\text { desviación típica }\end{array}$ & $t$ & $p$ & $d$ Cohen \\
\hline Autoexpresión en situaciones sociales (I) & $32,20(20,10)$ & $67,60(19,79)$ & $-10,01$ & 0,00 & $-1,775$ \\
Defensa de los propios derechos como consumidor (II) & $23,32(23,22)$ & $66,32(16,45)$ & $-8,52$ & 0,00 & $-2,137$ \\
Expresión de enfado o disconformidad (III) & $43,12(29,27)$ & $85,48(15,22)$ & $-7,06$ & 0,00 & $-1,816$ \\
Decir no y cortar interacciones (IV) & $41,36(25,73)$ & $77,20(15)$ & $-8,61$ & 0,00 & $-1,702$ \\
Hacer peticiones (v) & $29,20(21,82)$ & $72,64(15,87)$ & $-9,10$ & 0,00 & $-2,277$ \\
Iniciar interacciones positivas con el sexo opuesto (vI) & $47,60(28,61)$ & $77(14,79)$ & $-5,33$ & 0,00 & $-1,639$ \\
PDG & $28,40(17,48)$ & $86,64(12,63)$ & $-18,82$ & 0,00 & $-3,819$ \\
\hline
\end{tabular}

Nota. Elaboración propia. 
Tabla 5

Medias y (desviaciones típicas) del grupo control y experimental en el pos-test

\begin{tabular}{lccccc}
\hline \multicolumn{1}{c}{ Escalas } & G. Control & G. Experimental & & \\
\cline { 2 - 6 } & $\begin{array}{c}\text { Mediay } \\
\text { desviación típica }\end{array}$ & $\begin{array}{c}\text { Media y } \\
\text { desviación típica }\end{array}$ & $t$ & $p$ & $d$ Cohen \\
\hline Autoexpresión en situaciones sociales (I) & $31,04(26,67)$ & $67,60(19,79)$ & $-5,50$ & 0,00 & $-1,557$ \\
Defensa de los propios derechos como consumidor (II) & $26,16(16,59)$ & $66,32(16,45)$ & $-8,59$ & 0,00 & $-2,431$ \\
Expresión de enfado o disconformidad (III) & $45,36(29,32)$ & $85,48(15,22)$ & $-6,07$ & 0,00 & $-1,718$ \\
Decir no y cortar interacciones (IV) & $41,64(28,93)$ & $77,20(15)$ & $-5,45$ & 0,00 & $-1,543$ \\
Hacer peticiones (v) & $33,64(30,23)$ & $72,64(15,87)$ & $-5,71$ & 0,00 & $-1,615$ \\
Iniciar interacciones positivas con el sexo opuesto (vI) & $40,60(22,46)$ & $77(14,79)$ & $-6,76$ & 0,00 & $-1,916$ \\
PDG & $28,60(20,33)$ & $86,64(12,63)$ & $-12,12$ & 0,00 & $-3,429$ \\
\hline
\end{tabular}

Nota. Elaboración propia.

Al realizar el análisis estadístico de los datos, se compararon las puntuaciones de cada escala y el total de la EHS en el pos-test. Se evidencia que en el pos-test el grupo experimental muestra un promedio más alto en relación con el grupo control. Asimismo, la puntuación t obtenida para cada escala y para el total de la pos-test muestra que existen diferencias estadísticamente significativas, lo cual indica que se fortalecieron las respuestas de los jóvenes ante cada escala y el total del pos-test al concluir el programa de intervención. Nuevamente, se aprecia que la magnitud de estas diferencias fue muy grande (Cohen, 1988).

\section{Discusión}

El presente trabajo tuvo como finalidad analizar los efectos comportamentales de un programa de habilidades sociales en jóvenes de 14 a 18 años en situación de vulnerabilidad, de la Fundación Club Campestre de Bucaramanga. Para llevar a cabo el objetivo de la investigación se realizó una medición pre-test pos-test, lo cual permitió medir las habilidades sociales de cada joven antes y después del programa. Como resultado del programa se generaron cambios comportamentales en los jóvenes, ya que su repertorio de conductas socialmente adecuadas aumentó o se reforzaron conductas que tenían previo al programa, resultados similares a los obtenidos por Durán y Begoña (2008), Camacho (2005), González, Ampudia y Guevara (2012), y Arancibia y Peres (2007). En esta investigaciones se muestra que los programas de intervención de este tipo son efectivos, ya que atienden las habilidades básicas en los jóvenes, ofrecen un repertorio de respuestas conductuales para la adecuada resolución de situaciones sociales y, sin importar el género de los participantes, los resultados son eficaces a la hora de una intervención comportamental. Pero, por el contrario, en un programa realizado por Riso (1998), este propone que la efectividad del mismo es mayor con un grupo de hombres y mujeres, ya que se presentan situaciones de interacción espontáneas y se facilita recrear juegos de roles entre géneros; sin embargo, los resultados no arrojaron diferencias después de la intervención.

El pre-test de la escala de habilidades sociales, tanto en el grupo experimental como en el grupo control, presenta niveles de socialización similares en sus puntuaciones en cuanto a temas de intervención tales como espacio personal, inteligencia emocional, asertividad, discernimiento, manejo de la agresividad, capacidad de hacer y recibir cumplidos, habilidades interpersonales, empatía y toma de decisiones; es decir, inicialmente el grupo total de jóvenes de la fundación poseían pocas habilidades sociales en su repertorio de interacción con otras personas.

Con la aplicación de las pruebas se comprobó que el nivel de habilidades sociales del grupo control y el grupo experimental antes de la aplicación del programa fue bajo y demostraban dificultades en la interacción adecuada con sus pares. Uno de los factores que pueden explicar esta problemática es que dentro del núcleo familiar de todos los jóvenes de la fundación no se encuentra una o las dos figuras paternales, y el aprendizaje de las habilidades sociales se lleva a cabo por medio de los familiares más cercanos o sus primeras interacciones con el entorno. Por eso, autores como Segura y Bernabeu (2003) destacan la importancia de los padres en el entrenamiento de las 
habilidades sociales, resultando especialmente significativa debido a: (a) el lugar básico que ocupan en el desarrollo temprano de las actitudes y capacidades sociales de los hijos; (b) la interacción continua, constante y duradera con los hijos; (c) las numerosas ocasiones que se presentan, de forma natural, para aplicar reforzamiento y facilitar el aprendizaje o la modificación de conductas de tipo interpersonal; y (d) la enorme influencia que tienen en la generalización y el mantenimiento de las habilidades sociales que se puedan aprender en el ámbito escolar. En este sentido, Caballo y Verdugo (2005) exponen que "la familia es la que controla el ambiente social donde el niño se mueve, la que proporciona oportunidades, experiencias y la que le posibilita contactos con otros niños" (p. 25), de manera que esta es una función que cumplen los padres como entes formadores del menor.

Otros factores, como la etapa de desarrollo vital, en este caso la adolescencia, influyen en el desarrollo de las habilidades sociales debido a que, según Camacho (2005), se trata de un momento en el que, además de producirse un cambio físico y psicológico importante en los sujetos, "cobrarán gran valor todos los aspectos del ámbito de relaciones y de intercambio social” (p. 5). Además, otro factor que interviene en el desarrollo de las habilidades son las relaciones entre pares, en razón a que precisamente a esta edad los individuos empiezan a ser más independientes de su familia y buscan su identidad a través de la identificación en su grupo social de iguales.

El estilo de aprendizaje también tiene una importancia vital en el desarrollo de habilidades sociales. En este sentido, Bandura (1982) indica que los niños aprenden a comportarse, tanto por medio de la instrucción, como de la observación. Así, este autor destaca que existen ciertos factores que influyen sobre las habilidades sociales, a saber: (a) la necesidad de proveer a los niños con métodos para enfrentar aspectos internos de su vida social, incluyendo reducción de estrés, autocontrol y toma de decisiones; y (b) aplicar métodos de forma intensiva, tales como observación, representación o instrucción, para que así se pueda generar un proceso efectivo en el aprendizaje de estas conductas.

Ya que el aprendizaje de las habilidades sociales está ligado a diferentes factores en los jóvenes, y que en la mayoría de los casos no se presentan las circunstancias adecuadas para su desarrollo, una alternativa eficaz y práctica para el aprendizaje de las habilidades sociales son los programas de intervención, en la medida en que generan cambios comportamentales en los repertorios de respuesta de los jóvenes frente a las diferentes situaciones a las que se enfrentan.

Los resultados en el pre-test mostraron puntuaciones bajas en la EHS, tanto en el grupo control como en el grupo experimental, es decir, los jóvenes de la fundación poseen pocas habilidades sociales y presentan comportamientos similares ante las situaciones sociales que enfrentan diariamente.

Por otro lado, el pos-test arrojó resultados diferentes, ya que en el grupo experimental se evidenció un aumento en las puntuaciones de la EHS, confirmándose así nuestra hipótesis. En este sentido, Peres (2008) concluyó en una investigación que, al terminar el programa de intervención, el grupo experimental presentó un incremento en sus habilidades de expresión, defensa de los derechos propios, expresión del enfado y decir no. Es decir, como plantea Rico (2009), la diferencia entre los grupos se puede atribuir a la aplicación del programa de entrenamiento de habilidades sociales. En este sentido, los resultados evidencian la efectividad del programa para desarrollar habilidades sociales en esta población, incluyendo la disminución de patrones agresivos de comportamiento, la adquisición de habilidades para solución de problemas, reconocimiento y expresión de sentimientos, en concordancia con hallazgos anteriores (Dib y Sturmey, 2007; Sim et al., 2006; Sukhodolsky et al., 2005), y con relación a la efectividad de estos programas para que adolescentes desarrollen una mejor interacción con sus pares y con adultos.

Las habilidades sociales de los jóvenes aumentaron en la evitación de respuestas agresivas a situaciones desconocidas, capacidad de toma de decisiones asertivas, creación de un esquema de respuestas conductuales adecuadas, identificación de las emociones propias y de los demás, y capacidad de hacer y recibir cumplidos. En cambio, los jóvenes del grupo control obtuvieron puntuaciones similares al pre-test en la EHS.

Los resultados principales son los cambios comportamentales de los jóvenes del grupo experimental, ya que poseen un repertorio de estrategias que les permite desenvolverse en las diferentes situaciones en las que se ven envueltos, y son capaces de reconocer los espacios mínimos de interacción con las demás personas. En este orden de ideas, Daymut (2010) plantea que "ser respetuoso con el espacio personal de otras personas es una habilidad social" (p. 239) y permite generar redes de interacción social adecuadas. Según Zamora, Vega y Poncelis (s. f.), las habilidades 
de interacción social son esenciales para el desarrollo del ser humano, y su impacto se evidencia en el funcionamiento del individuo, así como en el manejo de la impulsividad y de respuestas agresivas.

Al mismo tiempo, dentro de los cambios comportamentales presentes en los jóvenes, Howard y Raymond (2012) se refieren a "la reducción de los niveles perjudiciales y excesivos de vivencia de la agresividad y de exteriorización de la agresividad" (p. 20), lo cual permite la convivencia pacífica entre los jóvenes en sus diferentes contextos. Otros efectos comportamentales son la capacidad de reconocer los logros de las personas, por eso Caballo (2000) define estas capacidades como conductas verbales específicas que resaltan características positivas de una persona, lo que genera cordialidad y buen trato entre los jóvenes. En este orden de ideas, otra característica desarrollada en el programa de intervención es la asertividad, definida por Llacuna y Pujol (2004) como la habilidad personal que nos permite expresar sentimientos, opiniones y pensamientos, en el momento oportuno, de la forma adecuada y sin negar ni desconsiderar los derechos de los demás.

Tras analizar los resultados obtenidos en el trabajo, se evidenció que existen diferentes investigaciones que llegaron a resultados similares respecto al cambio conductual generado en el grupo experimental (Inglés, 2009; Caballo y Verdugo, 2005; Camacho, 2005; Peres, 2008; Rico, 2009), en las cuales se recomienda como herramienta de intervención primaria los programas de habilidades sociales en los jóvenes con dificultades en el desarrollo de las mismas.

El presente estudio aportó datos en un grupo de jóvenes en situación de vulnerabilidad, lo cual sienta algunas bases para llevar a cabo estudios con poblaciones más amplias. Futuras investigaciones han de tener en cuenta las características individuales y del entorno físico y social, así como el impacto de otros factores psicosociales en los jóvenes.

El diseño de este estudio nos lleva a considerar la realización de investigaciones de tipo longitudinal en las cuales se puedan evaluar las habilidades sociales de los jóvenes con el paso del tiempo, así como la adquisición de estas habilidades y su relación con otras variables tales como personalidad, estilos de vida y variables psicológicas, entre otros.

Se sugiere incluir un programa de intervención que considere la relación de los jóvenes con padres, profesores y otros actores sociales, con el fin de suministrarles mayores oportunidades de desarrollo psicológico y social.

\section{Referencias}

Arancibia, G. y Peres, X. (2007). Programa de autoeficacia en habilidades sociales para adolescentes. AJAYU, 35 133-140.

Arkowitz, H., Hinton, R., Perl, J. y Himadi, W. (1978). Treatment strategies for dating anxiety in college men based on real-life practice. Counseling Psychologist, 7, 41-46.

Arvelo, L. (2003). Función paterna, pautas de crianza y desarrollo psicológico en adolescentes: implicaciones psicoeducativas. Acción Pedagógica, 12(1), 20-30.

Bandura, A. (1982). Teoría del Aprendizaje Social. Madrid, España: Escasa-Calpe.

Beelmann, A. y Lösel, F. (2006). Child social skills training in developmental crime prevention: Effects on antisocial behavior and social competence. Psicothema, 18(3), 603-610

Betina, A. y Contini, N. (2011). Las habilidades sociales en niños y adolescentes. Su importancia en la prevención de trastornos psicopatológicos. Fundamentos en $\mathrm{Hu}$ manidades, 12(23), 159-182.

Bradlyn, A. S., Himadi, W. G., Crimmins, D. B., Christoff, K. A., Graves, K. G. y Kelly, J. A. (1983). Conversational skills training for retarded adolescents. Behavior Therapy, 14, 314-325.

Caballo C. y Verdugo, M. (2005). Habilidades sociales. Programa para mejorar las relaciones sociales entre niños y jóvenes con deficiencia visual y sus iguales sin discapacidad. Organización Nacional de Ciegos Españoles, 23, 25-28.

Caballo, V. (2000). Manual de Evaluacion y Entrenamiento de las Habilidades Sociales. Madrid, España: Siglo XXI.

Caballo, V. (2009). Manual de técnicas de terapia y modificación de conducta. México: Siglo Xxi.

Camacho, C. M. (2005). Habilidades sociales: un programa de intervencion. Revista Profesional Española de Terapia Cognitivo-Conductual, 3, 2-25.

Clark, K. y Kirisci, L. (1996). Posttraumatic stress disorder, depression, alcohol use disorders and quality of life of adolescents. Anxiety, 2, 226-33.

Cohen, J. (1988). Statistical power analysis for the behavioral sciences (2nd Ed.). Hillsdale, NJ: Erlbaum.

Conger, J. y Conger, A. (1982). Components of heterosocial competence. New York: Guilford Press.

Cuervo, A. (2010). Pautas de crianza y desarrollo socioafectivo en la infancia. Perspectiva Psicológica, 6(1), 111-121. 
Daymut, J. (2010). El Espacio Personal-Una Habilidad Social. Handy Handouts, 48, 239-240.

Dib, N. y Sturmey, P. (2007). The effects of verbal instruction, modeling, rehearsal, and feedback on correct posture during flute playing. Behavior Modification, 31(4), 382-388.

Durán, L. y Begoña, M. (2008). Programa de habilidades sociales. Miembros del Dpto. de Orientación del IESO de "La Parra", 1-5.

Englander-Golden, P., Elkonin, J. y Satir, V. (1986). Brief 'Say it straight' training and follow up in adolescent substance abuse prevention. J Primary Prevention, 6, 219-30.

Fernández-Daza, M. P. y Fernández-Parra, A. (2013). Problemas de comportamiento y competencias psicosociales en niños y adolescentes institucionalizados. Universitas Psychologica, 12(3), 797-810.

Francis G., Last, C. G. y Strauss, C. C. (1992). Avoidant personality disorder and social phobia in children and adolescents. Journal of the American Academy of Child and Adolescent Psychiatry, 3(6), 1086-1089

Gilchrist, L. D., Schinke, S. P., Trimble, J. E. y Cvetkovich, G. T. (1987). Skills enhancenebt to prevent substance abuse among American Indian adolescents. International Journal of the Addictions, 22, 869-879.

Gismero, E. (2000). EHs. Escala de habilidades sociales. Madrid: TEA ediciones .

González, C., Ampudia, A. y Guevara, Y. (2012). Programa de intervención para el desarrollo de habilidades sociales en niños institucionalizados. Acta colombiana de Psicología, 5, 44-47.

González, L. y Méndez, L. (2006). Relación entre autoestima, depresión y apego en adolescentes urbanos de la comuna de Concepción, Chile. Terapia Psicológica, 24(001), 5-14.

González, I., Valdés, J., Domínguez, A., Palomar, J. y González, S. (2008). Características psicosociales en niños en contexto de riesgo y de no riesgo. Persona, 11, 92-107.

Gracia, E., Lila, M. y Musitu, G. (2005). Rechazo parental y ajuste psicológico y social de los hijos. Salud Mental, 28 (2), 73-81.

Howard, K. y Raymond, C. (2012). El manejo de la agresividad. Manual de tratamiento completo para profesionales. Nueva York: McGraw-Hill.

Inglés, C. J. (2009). Enseñanza de habilidades interpersonales para adolescentes: Programa Pehia. Madrid: Ediciones Pirámide.
Inglés, C. J., Delgado, B., Bautista, R., Torregrosa, M. S., Espada, J. P. y García-Fernández, J. M. (2007). Factores psicosociales relacionados con el consumo de alcohol y tabaco en adolescentes españoles. Internacional Journal of Clinical and Health Psychology, 7(2), 403-420.

Inglés, C. J., Méndez, X. e Hidalgo, M. D. (2001). Dificultades interpersonales en la adolescencia: ¿factor de riesgo de fobia social? Revista de Psicopatología y Psicología Clínica, 6(2), 91-104.

Inglés, C. J., Piqueras, J. A., García-Fernández, J. M., García-López, L. J., Delgado, B., y Ruiz-Esteban, C. (2010). Diferencias de género y edad en respuestas cognitivas, psicofisiológicas y motoras de ansiedad social en la adolescencia. Psicothema, 22(3), 376-381.

La Greca, A. M. y López, N. (1998). Social anxiety among adolescents: Linkages with peer relations and friendships. Journal of Abnormal Child Psychology, 26, 83-94.

Lawrence, B. y Bennet, S. (1992). Shyness and education: The relationship between shyness, social class and personality variables in adolescents. British Journal of Educational Psychology, 62, 257-263.

Lazarus, A. y Folman, E. (1971). Behavior therapy and beyong. Nueva York: McGraw-Hill.

Lemanek, K., Williamson, D., Gresham, F. y Jensen, B. (1986). Social skills training with hearing-impaired children and adolescents. Behaviour Modification, 10(1), 55-71.

Liberman, R. (1975). Personal Effetiveness. New York: Research Press.

Llacuna, J. y Pujol, L. (2004). La conducta asertiva como habilidad social. Barcolena: Albor-Cohs.

Martínez, A. E., Inglés, C. J., Piqueras, J. A. y Ramos, V. (2010). Importancia de los amigos y los padres en la salud y el rendimiento escolar. Electronic Journal of Research in Ecucational Psychology, 8(11), 111-138.

Méndez, F. X., Inglés, C. J. e Hidalgo, M. D. (2002). Estrés en las relaciones interpersonales: Un estudio descriptivo en la adolescencia. Ansiedad y Estrés, 8, 23-31.

Michenbaum, D., Butler, L. y Gruson, L. (1981). Competencia social. Nueva York: Guilford Press.

Milchelson, L., Sugai, D., Wood R. y Kazdin, A. (1987). Las habilidades sociales en la infancia: evaluación y tratamiento. Barcelona: Martínez Roca

Ministerio de Educación Nacional (2005). Lineamientos de política para la atención educativa a poblaciones vulnerables. Recuperado de http://www.oei.es/quipu/colombia/politica_vulnerables.pdf 
Meyer, A. y Farrell, A. (1998). Social Skills Training to Promote Resilience in Urban Sixth Grade Students: One Product of an Action Research Strategy to Prevent Youth Violence in High-Risk Environments. Education and Treatment of Children, 21(4), 461-488.

Moore, S. G. (1997). The role of parents in the development of peer group competence. Washington, DC: Eric Digest.

Nitkowski, D., Petermann, F., Büttner, P., Krause, C. y Petermann, U. (2009). Behavior modification of aggressive children in child welfare: Evaluation of acombined intervention program. Behavior Modification, 33(4), 474-492.

Oliva, A., Parra, A. y Arranz, E. (2008). Estilos relacionales parentales y ajuste del adolescente. Infancia y Aprendizaje, 31(1), 93-106.

Phillips, L. y Zigler, E. (1961). Social competence: The action-thought parameter and vicariousness in normal and pathological behaviors. Journal of Abnormal and social psychology, 13, 137-146.

Openshaw, D. K., Mills, T. A., Adams, G. R. y Durso, D. D. (1992). Conflict resolution in parent-adolescent dyads: The influence of social skills training. Journal of Adolescent Research, 7, 457-468.

Peña, M., Maiquez, M. L. y Rodrigo, M. J. (2014). Efectos de la inclusión de contenidos de desarrollo personal en un programa de educación parental para familias en riesgo psicosocial. Anales de psicología, 30(1), 201-210.

Peres, M. (2008). Habilidades sociales en adolescentes institucionalizados para el afrontamiento de su entorno inmediato (Tesis doctoral), Universidad de Granada, Granada.

Pichardo, M. C., Justicia, F. y Cabezas, M. F. (2009). Prácticas de crianza y competencia social en niños de 3 a 5 años. Pensamiento Psicológico, 6(13), 37-48.

Plienis, A. J., Hansen, D. J., Ford, F., Smith, B. S., Stark, L. y Kelly, J. A. (1987). Behavioral small group training to improve the social skills of emotionally-disordered adolescents. Behavior Therapy, 18, 17-32.

Rao, P. A., Beidel, D. C., Turner, S. M., Ammerman, T., Crosby, L. E. y Sallee, F. R. (2007). Social anxiety disorder in childhood and adolescence: Descriptive psychopathology. Behaviour Research and Therapy, 45, 1181-1191.

Rico, L. (2009). Creación de un programa de intervención en habilidades sociales para adolescentes de la Fundación Hogar Niño Jesús de Belén en San Gil (Trabajo de grado especialización). Universidad Pontificia Bolivariana, Bucaramanga, Colombia.
Redondo, J. e Inglés, C. J. (2009). Conducta prosocial. Atribuciones causales y rendimiento académico en adolescentes. San Juan de Pasto: I.U. Cesmag.

Riso, W. (1998). Entrenamiento asertivo. Medellín: Rayuela.

Rocha, A. (2003). El sistema de interacción familiar asociado a la autoestima de menores en situación de abandono moral o prostitución (Disertación doctoral no publicada). Universidad Nacional Mayor de San Marcos, Lima, Perú.

Salter, A. (1949). Terapia de reflejos condicionados. Nueva York: Guilford Press.

Savidge, C., Christie, D., Brooks, E., Stein, S. y Wolpert, M. (2004). A pilot social skills group for socially disorganized children. Clinical Child Psychology and Psychiatry, 9(2), 289-296.

Schinke, S. P., Blythe, B. y Gilchrist, L. (1981). Cognitive behavioral prevention of adolescent pregnancy. Journal of Counseling Psychology, 28(5), 451-454.

Schloss, P. J., Schloss, C. N. y Harris, L. (1984). A multiple baseline analysis of an interpersonal skill training program for depressed youth. Behavior Disorders, 9, 182-188.

Segura, D. y Bernabéu, M. (2003). Programa de entrenamiento a madres, padres y profesores en habilidades sociales. Murcia: Scout Asde.

Sim, L., Whiteside, S., Dittner, C. y Mellon, M. (2006). Effectiveness of a social skillstraining program with school age children: Transition to the clinical setting. Journal of Child and Family Studies, 15(4), 408-417.

Solís, A. y Montoya, J. (2007). Proceso de adaptación de personas costarricenses menores de edad ubicadas en adopción en España. Acta Colombiana de Psicología, 10(1), 111-122.

Spence, S., Donovan, C. y Brechman-Toussaint, M. (2000). The treatment of childhood social phobia: The effectiveness of a social skills training-based, cognitivebehavioral intervention, with and without parental involvement. Journal of Child Psychology and Psychiatry, 41(6), 713-726.

Sukhodolsky, D., Golub, A., Stone, E. y Orban, L. (2005). Dismantling anger control training for children: A randomized pilot study of social problem-solving versus social skills training components. Behavior Therapy, 36(1), 15-23.

Walters, K. S. e Inderbitzen, H. M. (1998). Social anxiety and peer relations among adolescents: testing a psychobiological model. Journal of Anxiety Disorders, 12, 183-198. 
Wolpe, J. (1958). Psychotherapy by Reciprocal Inhibition. California: Pergamon Press.

Zambrano, A., Muñoz, J. y González, M. (2012). Variables psicosociales del entorno comunitario asociadas a procesos de desadaptación social en adolescentes: reflexiones a partir de un estudio de caso. Universitas Psychologica, 11(4), 1135-1145.
Zamora, M. C., Vega, L. y Poncelis, M. F. (s. f.). Manual para promover el desarrollo de habilidades sociales en niños y niñas preescolares. Recuperado de: http://www. psicologia.unam.mx/contenidoEstatico/archivo/files/ Banners/manual-desarrollohabilidades-sociales.pdf 